\title{
Detecting factorization effect in continuous-time quantum walks
}

\author{
Jin-Hui Zhu' , Li-Hua Lu' ${ }^{1 *}$ (I) and You-Quan Li'
}

\author{
"Correspondence: Ihlu@zju.edu.cn \\ 'Zhejiang Province Key Laboratory \\ of Quantum Technology \& Device \\ and Department of Physics, \\ Zhejiang University, Hangzhou, \\ 310027, P.R. China
}

\begin{abstract}
We consider an continuous-time quantum walk on triple graphs with a potential well in one of the terminal sites on the main chain and study its dynamical properties in the open system by introducing a 'spontaneous damping' process between the terminal site and its nearest neighbour one. Calculating the stable probability of the terminal site and the time evolution of probabilities of the other sites on the main chain, we show that the system can manifest the factorization and coherence protection phenomena when the length and position of the side chain satisfy some concrete conditions. Furthermore we investigate the effect of disorder on the hopping strength and show that the existence of the disorder can destroy the coherence protection phenomena and increase the stable probability of the terminal site on the main chain.
\end{abstract}

Keywords: Quantum walk; Factorization effect; Disorder

\section{Introduction}

Since quantum walk was proposed as the quantum mechanical counterpart of the classical random walk [1], it has received increasing attention in recent years due to its potential applications in many fields. For example, the quantum walk can not only provide us a simple model for study of coherent quantum effect in physical systems [2] but also offer us an advanced tool for quantum simulations [3, 4] and quantum algorithms [5-7]. As we know, unlike the classical random walk that is formulated by the evolution of probability, the quantum walk is formulated by the evolution of probability amplitude. Then in comparison to classical random walk, the quantum walk will diffuse faster than the classical random walk due to the existence of interference effects, which implies that the quantum walk has more advantages at certain search algorithms $[6,8,9]$.

According to the type of walking, the quantum walk can be divided into two main classes: discrete-time quantum walk and continuous-time quantum walk. Unlike the discrete-time quantum walk, the continuous-time quantum walk [10] can be realized through continuous tunneling between neighbour sites but does not need a quantum coin to generate a superposition state for each step. So the continuous-time quantum walk can be implemented in many conventional lattice system and easily used to simulate some quantum effects of those lattice systems. For example, we found the switching effect of

(c) The Author(s) 2021. This article is licensed under a Creative Commons Attribution 4.0 International License, which permits use sharing, adaptation, distribution and reproduction in any medium or format, as long as you give appropriate credit to the original author(s) and the source, provide a link to the Creative Commons licence, and indicate if changes were made. The images or other third party material in this article are included in the article's Creative Commons licence, unless indicated otherwise in a credit line to the material. If material is not included in the article's Creative Commons licence and your intended use is not permitted by statutory regulation or exceeds the permitted use, you will need to obtain permission directly from the copyright holder. To view a copy of this licence, visit http://creativecommons.org/licenses/by/4.0/. 
the side chain on quantum walks on triple graphs in our early work [11] where we did not focus the dynamical features of each site but only investigated the interchange of probabilities between the two parts of main chain for a closed system. As we aware, the dynamical features of each site on continuous-time quantum walks are also fascinating which are mainly determined by the type of graph for closed systems [12-22], whereas, for open systems the propagation features are affected not only by the type of graph but also by the coupling with the environment. Therefore, in this paper we construct an open quantumwalk system on a triple graph with a potential well in one of the terminal sites on the main chain and study its dynamical properties. We calculate the stable probability of the terminal site and the time evolution of probabilities of each point on the left part of the main chain, and find that the stable probability of the terminal site can sensitively reflect the relation between the length and the position of the side chain. The system can manifest the factorization and coherence protection effect when the length and the position of the side chain satisfy some concrete conditions. Furthermore, we investigate the effect of disorders on hopping strength and show that the existence of disorders can destroy that factorization and coherence protection effect. The results exhibit the potential application of the continuous-time quantum walk on the quantum simulation and are expected to enlighten the design of new detection advices.

This paper is organized as follows. In the next section, we model the continuous-time quantum walk system on a triple graph and give the Lindblad equation with considering a 'spontaneous damping' process between the terminal site with a potential well and its nearest neighbour one. In Sect. 3, with the help of the Lindblad equation we study the dynamical features of the system, and show that the system can exhibit the factorization and coherence protection phenomena when the length and position of the side chain satisfy some concrete conditions. In Sect. 4, we study the effect of disorders of hopping strength on the factorization and coherence protection phenomena and find that the existence of disorders can destroy the factorization and coherence protection phenomena. In the last section, we give a brief summary.

\section{Modelling quantum walk in open system}

We consider a continuous-time quantum walk of single particle on the following triple

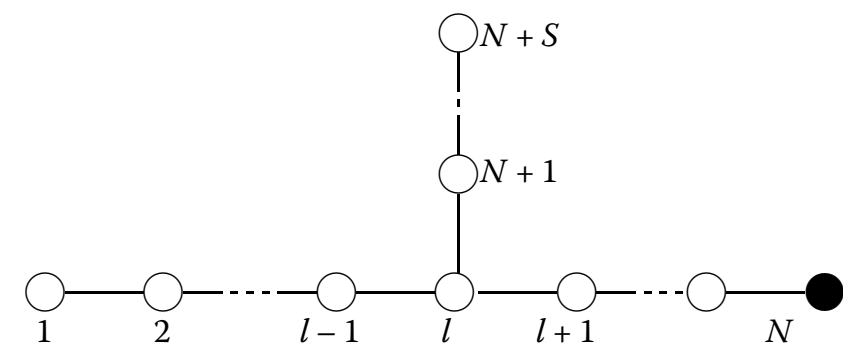

graph.

The graph contains a main chain of $N$ points labeled by $1,2, \ldots, N$, and a side chain of $S$ points labeled by $N+1, \ldots, N+S$, where the side chain is connected to the $l$ th point on the main chain. So the side chain divides the main chain into two parts, the left chain and the right chain. There is a potential well in the $N$ th point which is noted in solid circle on the above graph. For convenience, the $N$ th point is called as sensor point in the following discussion because its stable probability can sensitively reflect the relation of the 
position and the length of the side chain, i.e., the relation of the two integers $l$ and $S$. Then the Hamiltonian describing the continuous-time quantum walk on such a graph can be written as

$$
\begin{aligned}
H= & \left(-J \sum_{j=1}^{N-1}|j\rangle\left\langle j+1\left|-J \sum_{j=N+1}^{N+S-1}\right| j\right\rangle\langle j+1|\right. \\
& +J|l\rangle\langle N+1|+\gamma| N\rangle\langle N|)+ \text { h.c. }
\end{aligned}
$$

where $|j\rangle$ denotes a state that the particle occupies the $j$ th point on the graph, $J$ the hopping strength between the nearest points, and $\gamma$ the potential energy of the sensor point.

Since there is an energy difference between the sensor point and its nearest neighbour one, it is reasonable to introduce an extra 'spontaneous damping' from the state $|N-1\rangle$ to the state $|N\rangle$. The 'spontaneous damping' can cause the particle in the $(N-1)$ th point to drop down into the sensor point emitting a piece of energy. Then the dynamical property of the quantum walk we considered can be determined by the Lindblad master equation $[23,24]$

$$
\begin{aligned}
\frac{\mathrm{d}}{\mathrm{d} t} \rho(t)= & -i[H, \rho(t)]+\lambda\left(2 L \rho(t) L^{\dagger}\right. \\
& \left.-L^{\dagger} L \rho(t)-\rho(t) L^{\dagger} L\right),
\end{aligned}
$$

where $\rho(t)=\sum_{j, k} \rho_{j k}|j\rangle\langle k|$ with $j, k=1,2, \ldots, N, N+1, \ldots, N+S$ denotes the density matrix of the system, $L$ the lindblad operator to characterize the 'spontaneous damping', and $\lambda=\frac{\gamma^{2}}{2\left(\gamma^{2}+1\right)}$ the 'spontaneous damping' strength. Here the matrix elements of the Lindblad operator $L$ are zeros except for $L_{N-1, N}=1$ because there is only one energy difference between the sensor point and its nearest neighbour one. Now we construct a quantum-walk system coupling with a certain environment by considering the 'spontaneous damping' process between the senor point and its nearest neighbour one.

We know that the existence of the side chain can make the quantum-walk system exhibit some novel phenomena, such as switching effect [11], even if the quantum walk on the triple graph is considered in a closed system. Note that considering the 'spontaneous damping' process between the senor point and its nearest neighbour one equals to construct a quantum-walk system coupling with a certain environment, which must make the system exhibit more fascinating propagation properties. In the following discussion, our main purpose is to find how the length and position of the side chain influence the stable probability of the sensor point. And we further expect that such an open quantum-walk system can be used to simulate the relation between two integers (i.e., $l$ and $S$ ), which maybe helpful for new detection devices.

\section{Dynamical features and factorization effect}

Let us assume that the particle is located in the first point on the main chain at the initial time and solve the master equation (2) numerically to investigate the dynamical features of the system. The results show that the probability of the sensor point will reach the stable value that depends on both the position and the length of the side chain. Whereas, the probabilities of certain points on the left chain may always oscillate with time when the 
position and the length of the side chain satisfy some concrete conditions, which implies that the existence of the side chain may protect the coherence of the system.

\subsection{The case of even I}

Now we are in the position to consider the case that the side chain is connected to the point of even number on the main chain (i.e., $l$ is even). In this case, the stable probability of the sensor point is affected obviously by the ratio of $l$ and $S+1$. Taking $l=6$ as an example, we plot time evolutions of probability of the sensor point for different $S$ in Fig. 1. From this figure, we can see that the stable probability of the sensor point for the case of $\frac{l}{S+1}$ being an integer is smaller than that for the other case (i.e., $l$ is not divisible by $S+1$ ). When $l$ is divisible by $S+1$, ie, $\frac{l}{S+1}$ is an integer, the larger the value of $\frac{l}{S+1}$, the larger the stable probability of the sensor point is. And for the case of $\frac{l}{S+1}$ being not an integer, if $l$ and $S+1$ have common divisors larger than 1 , the stable probability of the sensor point is smaller than that for the case that $l$ and $S+1$ have no common divisors larger than 1 .

Except for the probability of the sensor point, the time evolution of probabilities of points on the left chain is also investigated. First let us see the case of $\frac{l}{S+1}$ being integers whose corresponding results are plotted in Fig. 2. From Fig. 2 (a) and (b), we can find that for the case of $S=5$ all the probabilities of points on the left chain always oscillate with time, and for the case of $S=2$ the probabilities $\left(P_{1}, P_{2}, P_{4}\right.$ and $\left.P_{5}\right)$ of the points 1 , 2,4 , and 5 oscillate with time but the probability $P_{3}$ can reach a very small stable value. Note that for such two cases the phases of the oscillations are not the same, and the phase difference is about $\pi$. This implies that the particle is difficult to propagate to the sensor point due to the interference cancellation, which confirms the results in Fig. 1 where the stable probabilities of the sensor point for $S=2$ and $S=5$ are smaller than that for the other $S$. Whereas, for the case of $S=1$ shown in Fig. 2 (c), the probabilities of all points on the left chain can reach stable values and there is no interference cancellation, so the stable probability of the sensor point for $S=1$ is larger than that for $S=2$ and $S=5$. In Fig. 3, we plot the time evolution of probabilities of points on the left chain for the case of $\frac{l}{S+1}$ being not integers. For such a case, we can see that the probabilities of all points on the left chain can reach stable values no matter what the value of $S$ is. Meanwhile, for the case of $l$ and $S+1$ having no common divisors larger than 1 (i.e., the case in Fig. 3 (a)) the probability of points on the left is much smaller than that for the case of $l$ and $S+1$ having common divisors larger than 1 (i.e., the case in Fig. 3(b)). This confirms that the

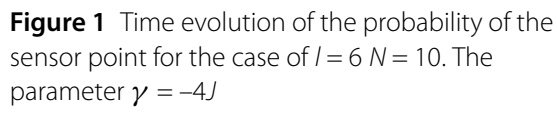



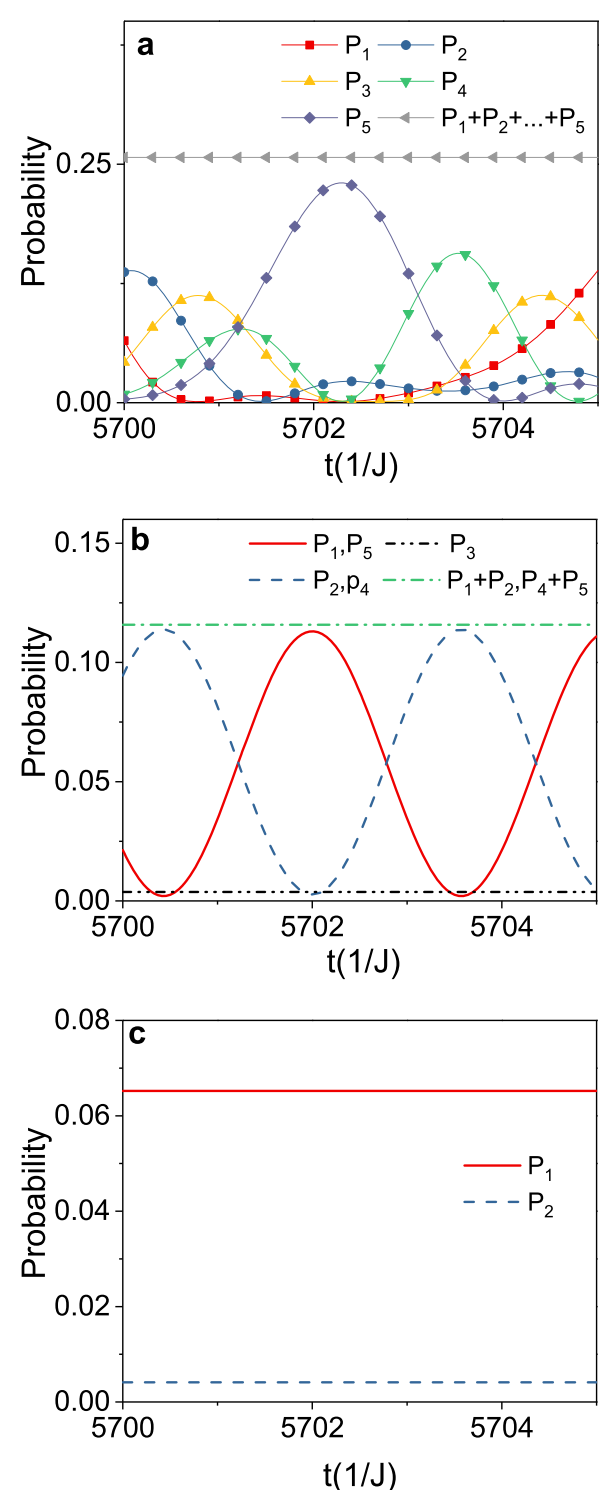

Figure 2 Time evolution of the probability of the points on the left chain after the probability of the sensor point reaches its stable value for the case of $I=6, S=5(\mathbf{a}), I=6, S=2(\mathbf{b})$, and $I=6, S=1$ (c) . The parameter is $\gamma=-4 J$
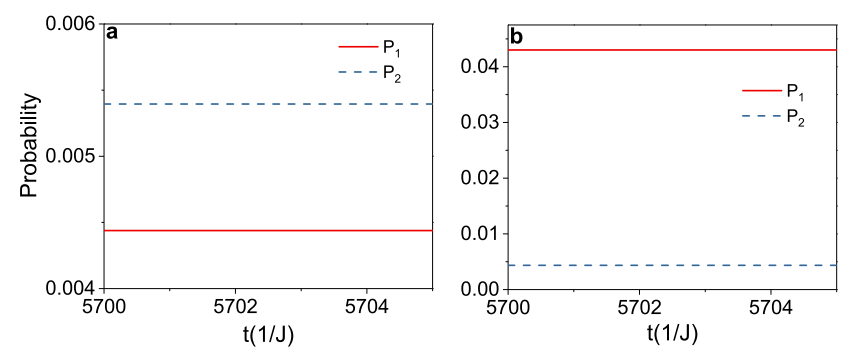

Figure 3 Time evolution of the probability of the points on the left chain after the probability of the sensor point reaches its stable value for the case of $I=6, S=4(\mathbf{a})$, and $I=6, S=3(\mathbf{b})$. The parameter is $\gamma=-4 \mathrm{~J}$ 
stable probability of the sensor point for the case of $S=3$ is smaller than that for the case of $S=4$ given in Fig. 1.

According to the above discussion, we know that for the side chain of certain sides the probabilities of some points on the left chain always remain oscillation although the 'spontaneous damping' is considered. Such oscillation depresses the propagation of the particle to the sensor point due to the interference cancellation. In order to characterize the dependence of the stable probability of the sensor point on the side chain, we introduce the concept of point kernel. The probability summation of the points in the point kernel can reach a stable value although the probability of each point in the point kernel always oscillates with time. The results are shown that the existence of the point kernel can depress the stable probability of the sensor point and the more points the point kernel contains, the smaller the stable probability of the sensor point is. The graphs describing the point kernel are plotted in the appendix. From Fig. 7, we can find that the graph can form point kernel with $S$ points for $\frac{l}{S+1}$ being integers, which is marked with dash rings. Whereas, if $l$ is not divisible by $S+1$, the point kernel with only one point can be formed for the case of $l$ and $S+1$ having common divisors larger than 1 but there are no point kernels for the case of $l$ and $S+1$ having no common divisors larger than 1 (see Fig. 8 in the appendix). This is agreeable with the results in Fig. 1.

\subsection{The case of prime I}

For the case of the side chain connecting to the point of prime number on the main chain (i.e., $l$ is prime), the probability of the sensor point can exhibit different features in comparison to the case of $l$ being even. In this case, taking the case of $l=7$ as an example, we plot the time evolution of the probability of the sensor point for the side chain with different number of points in Fig. 4. From this figure, we can see that the stable probability of the sensor point is always large and that almost does not change with the value of $S$ except for the case of $l=S+1$.

In Fig. 5, we plot the time evolution of probabilities of the points on the left chain for the cases of $S=6$ and $S=2$. From Fig. 5 (a), we can find that for the case of $l=7$ and $S=6$ the probabilities of all points on the left chain oscillate with time and their summation remains stable when the probability of the sensor point reaches its stable value. For this case, all the points on the left chain form a point kernel (see Fig. 9 (a)). Just like the discussion in Sect. 3.1, the interference cancellation of the probability oscillations makes the particle

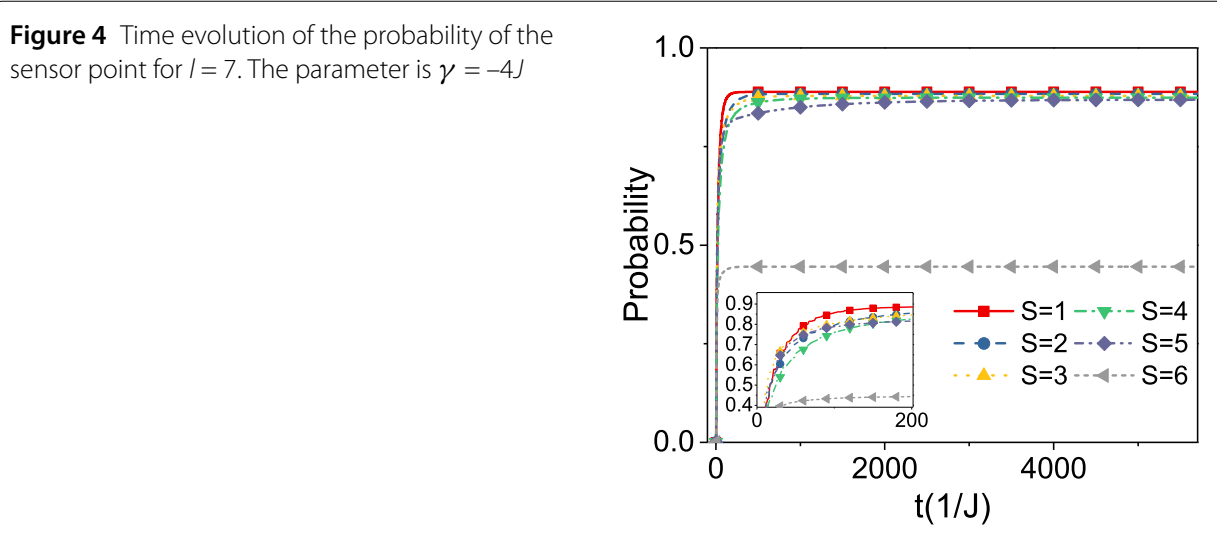



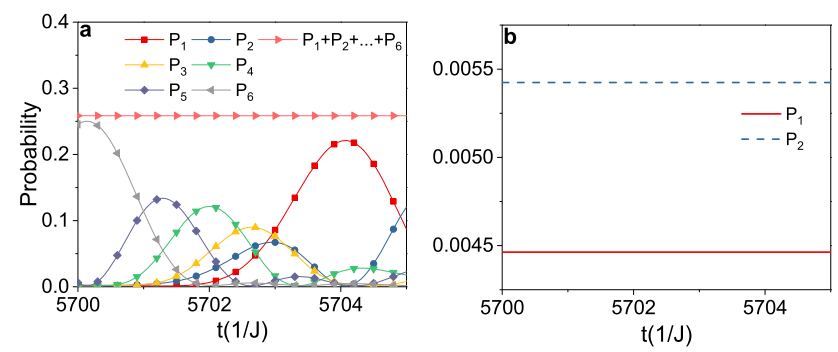

Figure 5 Time evolution of probabilities of the points on the left chain after the probability of the sensor point reaches its stable value for the case of $I=7, S=6(\mathbf{a})$ and $I=7, S=2(\mathbf{b})$. The parameter is $\gamma=-4 \mathrm{~J}$

difficult to propagate to the sensor point. Whereas, for the other cases, i.e., $l \neq S+1$, there is no point kernel formed in the graph so that the probabilities of all points on the left chain can reach stable values such as the results shown in Fig. 5 (b). So the stable probability of the sensor point for the case of $l=S+1$ is obviously smaller than that for the other cases, i.e., $l \neq S+1$. According to the above discussion, we can conclude that the stable probability of the sensor point is obviously affected by $\frac{l}{S+1}$ no matter whether $l$ is even or prime. The graph can always form point kernel for both even $l$ and prime $l$ when $\frac{l}{S+1}$ is integer. For this case, the stable probability of the sensor point is much smaller than the case of $l$ being not divisible by $S$ because the interference cancellation of probability oscillations of the points on the left chain makes the particle difficult to propagate to the sensor point. Note that in comparison to the case of even $l$, for the case of prime $l$ the graph can not form point kernel with only one point (see Fig. 9 (b)) when $\frac{l}{S+1}$ is not integer. This confirms the results in Fig. 4 where is shown that the stable probability of the sensor point does not change with the value of $S$ except for the case of $l=S+1$.

\section{Hopping strength disorder}

We know that random disorders in quantum walk can bring in the fascinating theoretical effects [25-27], and can also provide additional tools in constructing quantum walks for particular applications [28]. Now we will investigate the effect of random disorders on the stable probability of the sensor point by introducing a random fluctuation into the hopping strength, i.e., $J=\bar{J}+\delta J$. Here $\bar{J}$ denotes the average value of the hopping strength and $\delta J$ the random fluctuation with $\delta J \ll \bar{J}$. We randomly choose a large number of $\delta J$ and calculate the time evolution of the density matrix $\rho(t)$ for each random $\delta J$, respectively, and then obtain the average time evolution of the density matrix $\bar{\rho}(t)$ by calculating the average value of that $\rho(t)$. Some results are plotted in Fig. 6.

In Fig. 6, we plot the dependence of the stable probability of the sensor point on the number of points of the side chain for $l=8$ and $l=7$. From Fig. 6, we can see that the existence of the disorder can make the stable probability of the sensor point become larger obviously if $\frac{l}{S+1}$ is an integer with $S$ larger than 1, but the effect of the disorder on the stable probability of the sensor point is negligible for the other cases. From the discussion in the above section, we know that for the case of $\frac{l}{S+1}$ being integers and $S$ larger than 1 , the system can not be decoherent completely if the disorder is not considered. Then the probability of some points always remains oscillation which can depress the stable probability of the sensor point due to their interference cancellation. Once the random disorder on the hopping strength is considered, it can destroy the remained coherence and the interference 

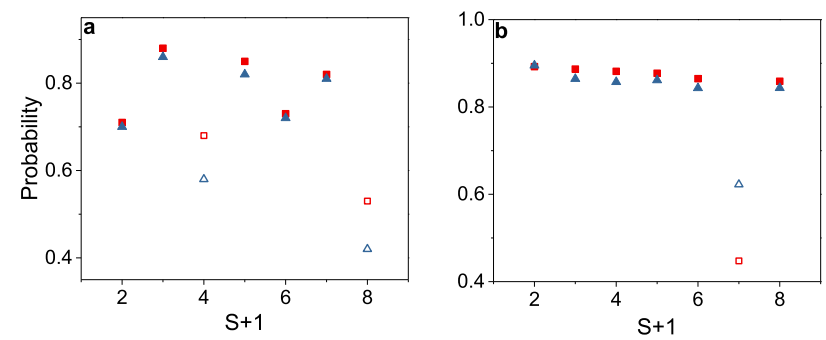

Figure 6 Dependence of the stable probability of the sensor point on the number of points of the side chain. The parameter is $I=8(\mathbf{a})$ and $I=7(\mathbf{b})$. The triangle refers to the results for the case of considering disorder and the square to the results for that of no disorder. The hollow triangle and square refer to the case of $\frac{1}{5+1}$ being integers with $S$ larger than 1

cancellation disappears, then the particle become easier to propagate to the sensor point whose stable probability becomes larger. Whereas, for the other cases the system can be completely decoherent by the 'spontaneous damping' even if there is no disorder on the hopping strength considered. So the decoherence effect of the disorder does not exhibit and the stable probability of the sensor point can not be changed obviously.

\section{Conclusion and discussion}

We considered a continuous-time quantum walk on a triple graph where there is a potential well on the last point (called as sensor point) on the main chain. Then we constructed an open quantum-walk system coupling with a certain environment by considering a 'spontaneous damping' between the sensor point and its nearest neighbour one. We studied the dependence of the length and position of the side chain on the stable probability of the sensor point, and showed that one can simulate the relation of two integers by the continuous-time quantum walk on a triple graph we considered. Additionally, we studied the time evolution of probabilities of the points on the left chain and found that the existence of the side chain can protect the coherence of the system for $\frac{l}{S+1}$ being integer and $S$ larger than 1 . So that the quantum-walk system always remains partial coherence even if we considered the 'spontaneous damping' process. For such a case, the particle is difficult to propagate to the sensor point due to the interference cancellation, so the stable probability of the sensor point is smaller obviously than that for the other cases. Such a coherence protection effect of the side chain can affect the stable probability of the terminal site obviously and may be used to increase the coherence time in quantum simulation. This can be analogous to that the entanglement protection effect can improve the efficiency of quantum simulation in digital quantum computing paradigm [29] that has more advantages in quantum error correction. Note that in Ref. [30] the authors showed that the quantum-walk system can be used to construct an X-gate of a single qubit as well as a control gate of two qubits in some concrete conditions, which implies that the quantum walk has relationship with the digital-analog quantum simulations. Meanwhile, we investigated the effect of the disorder on the hopping strength, and showed that the existence of the disorder can obviously increase the stable probability of the sensor point if $\frac{l}{S+1}$ is an integer and $S$ larger than 1, but the effect of the disorder on the stable probability of the sensor point is tiny for the other cases. In a word, we studied the dynamical properties of the continuous-time quantum walk on a triple graph and showed that such a system 
can be used to detect the factorization effect. The results are expected to enlighten the design of new detection devices which are based on the quantum algorithm but not on the classical one.

Since the open quantum-walk system we considered can exhibit the above novel features, we expect that can be observed in experiment and even can lighten one to design detection devices. As we know the quantum walk has been realized in many systems, such as cold atoms [31], superconducting circuits [32] and photons [33] where the quantum walk were investigated without the coupling with the environment. Whereas, in Ref. [34, 35] the quantum walk with decoherence was studied experimentally in cold atom and photon system, respectively, which implies that the open quantum-walk system we constructed theoretically may be realized in experiment.

\section{Appendix: Graphs describing the shape of point kernels}

Graphs describing the shape of point kernel are plotted, where the point kernel is marked with the dash ring.

For $\frac{l}{S+1}$ being integers with even $l$, the graph can form point kernels with $S$ points. In Fig. 7, we plot an example of $l=6$ and $S=1,2,5$, which is shown that the two nearest point kernels on the left chain must be separated by a point with stable probability, such as the third point in Fig. 7 (b), and the connection point $l$ must be adjacent to a point kernel on the main chain and side chain, respectively. Note that for the case of $S=1$, we think that the graph forms point kernel with only one point. Due to the fact that the total probability of points in the point kernel should reach a stable value, the probabilities of all points on the left chain can reach stable values although $\frac{l}{S+1}$ is integer (see Fig. 2 (c)). In Fig. 8, we plot the shape of point kernels for the case of $\frac{l}{S+1}$ being not integers with $l=6$. Here for $S=4$, i.e., $l$ and $S+1$ have no common divisors larger than 1 , the graph can not form point kernel, but for $S=3$, the graph can form point kernel with one point.

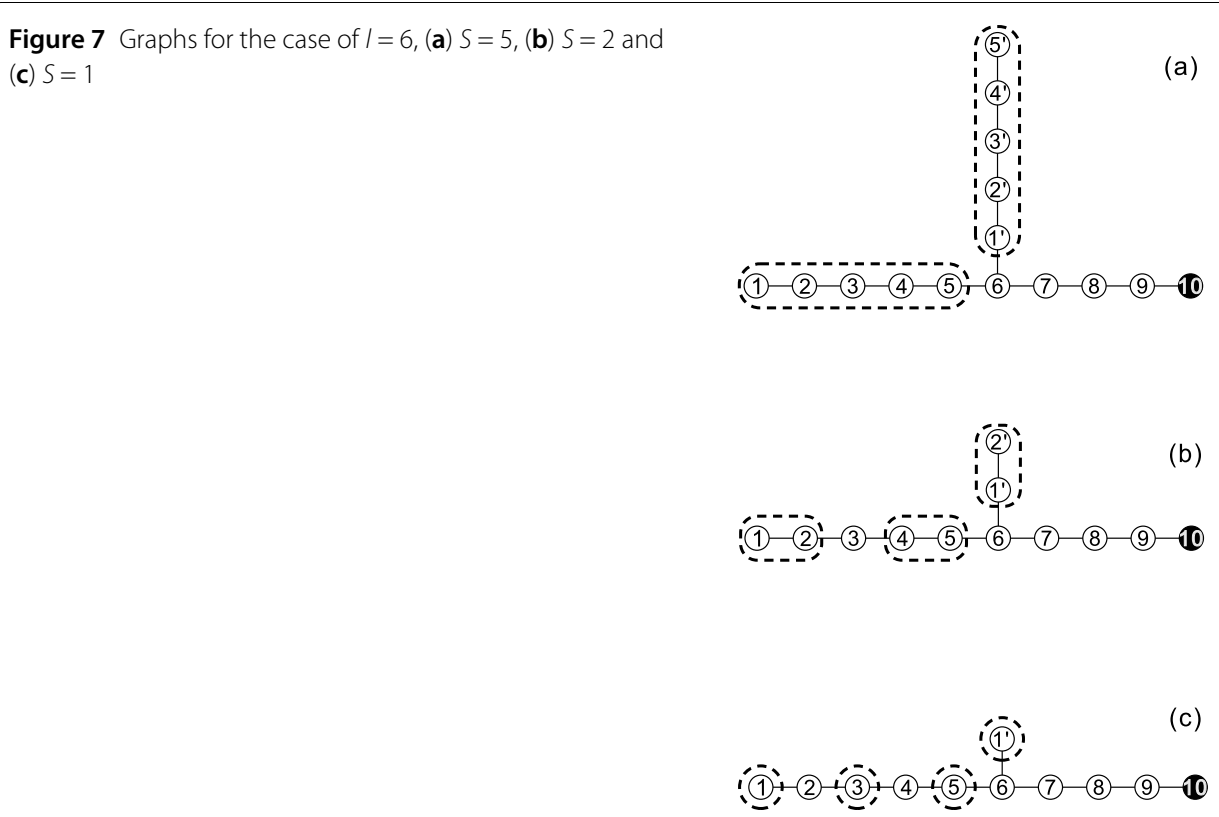



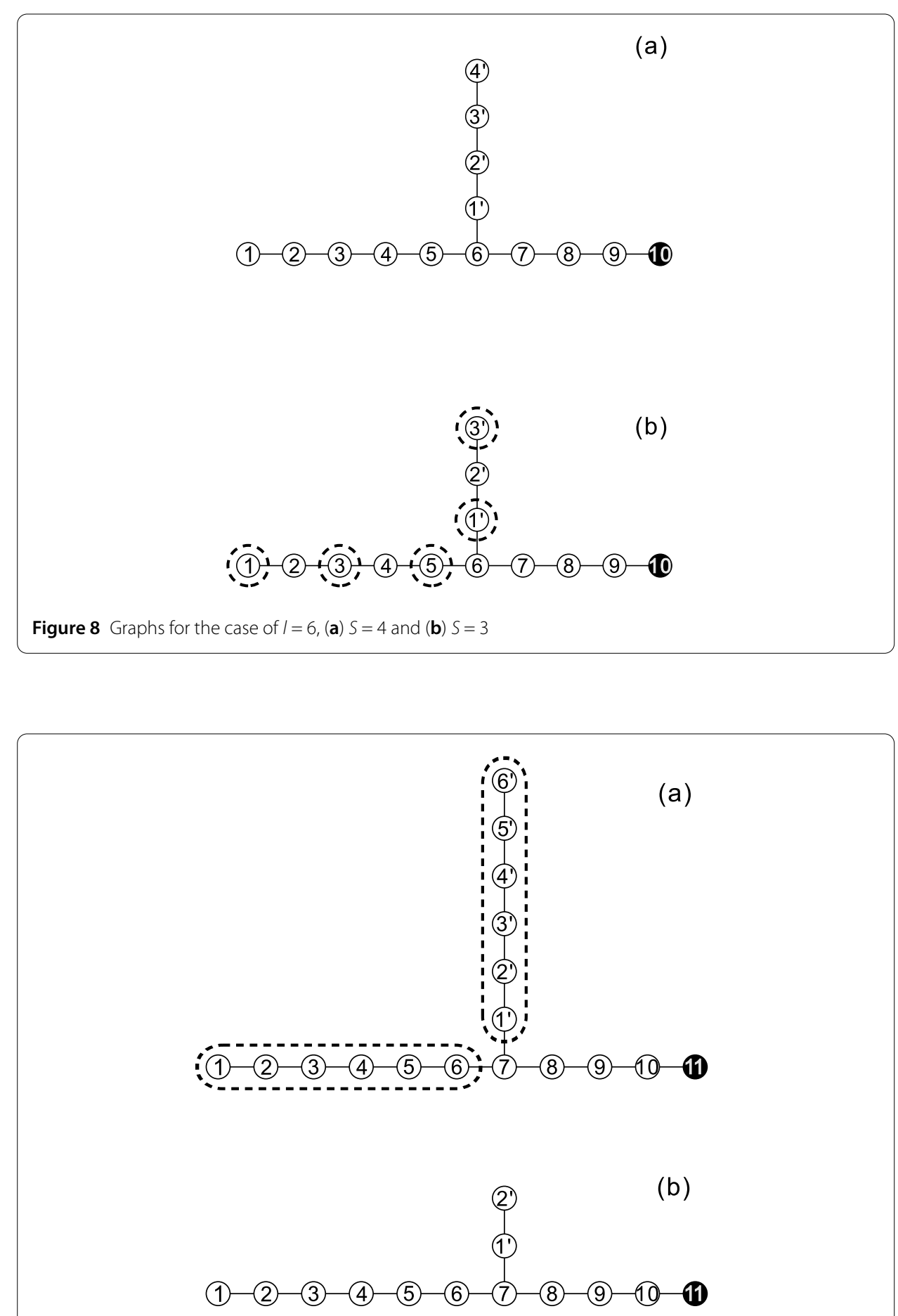

Figure 9 Graphs for the case of $I=7$, (a) $S=6$ and (b) $S=2$

For the case of prime $l$, the graph can form point kernel with $S$ points only when $l=S+1$. Taking $l=7$ as an example, we plot the graph describing the shape of point kernels in Fig. 9 which is shown that for $S=6$ the graph can form point kernel with 6 points but for $S=2$ there is no point kernels formed. 


\section{Acknowledgements}

The authors acknowledge the supporting of the NKRDP of China (2017YFA0304304) and the National Natural Science Foundation of China (Grant No. 11935012).

\section{Funding}

This work was supported by the NKRDP of China (2017YFA0304304) and the National Natural Science Foundation of China (Grant No. 11935012)

\section{Abbreviations \\ Not applicable.}

\section{Availability of data and materials}

The data sets supporting the results of this article are included within the article and Github.

\section{Competing interests}

The authors declare that they have no competing interests.

\section{Authors' contributions}

$J-H Z, L-H L$ and $Y-Q L$ together conceived the idea for the study. J-HZ performed the numerical calculation and plotted the figures. All the authors discussed and got the conclusion of the manuscript. J-HZ and L-HL wrote the manuscript. All authors reviewed the manuscript. All authors read and approved the final manuscript.

\section{Publisher's Note}

Springer Nature remains neutral with regard to jurisdictional claims in published maps and institutional affiliations.

Received: 13 July 2020 Accepted: 22 April 2021 Published online: 03 May 2021

\section{References}

1. Aharonov Y, Davidovich L, Zagury N. Quantum random-walks. Phys Rev A. 1993;48:1687.

2. Pfister G, Scher H. Dispersive (non-Gaussian) transient transport in disordered solids. Adv Phys. 1978;27:747.

3. Kempe J. Quantum random walks: an introductory overview. Contemp Phys 2003:44:307.

4. Hines AP, Stamp PCE. Quantum walks, quantum gates, and quantum computers. Phys Rev A. 2007:75:062371

5. Mohseni M, Rebentrost P, Lloyd S, Aspuru-Guzik A. Environment-assisted quantum walks in photosynthetic energy transfer. J Chem Phys. 2008:129:174106.

6. Shenvi N, Kempe J, Whaley RB. Quantum random-walk search algorithm. Phys Rev A. 2003;67:052307.

7. Venegas-Andraca SE. Quantum walks: a comprehensive review. Quantum Inf Process. 2012;1 1:1015.

8. Childs AM, Cleve R, Deotto E, Farhi E. Exponential algorithmic speedup by quantum walk. In: Proc. 35th ACM symp. on theory of computing. 2003.

9. Balu R, Liu CB, Venegas-Andraca SE. Probability distributions for Markov chain based quantum walks. J Phys A, Math Theor. 2018;51:035301.

10. Farhi E, Gutmann S. Quantum computation and decision trees. Phys Rev A. 1997:58:915.

11. Du YM, Lu LH, Li YQ. Switching effect of the side chain on quantum walks on triple graphs. Phys Rev A. 2015;92:012309.

12. Košík J, Bužek V. Scattering model for quantum random walks on a hypercube. Phys Rev A. 2005;71:012306.

13. Krovi H, Brun TA. Hitting time for quantum walks on the hypercube. Phys Rev A. 2006;73:032341,

14. Marquezino FL, Portugal R, Abal G, Donangelo R. Mixing times in quantum walks on the hypercube. Phys Rev A. 2008:77:042312

15. Makmal A, Zhu M, Manzano D, Tiersch M, Briegel HJ. Quantum walks on embedded hypercubes. Phys Rev A. 2014;90:022314.

16. Bednarska M, Grudka A, Kurzyński P, Łuczak T, Wójcik A. Quantum walks on cycles. Phys Lett A. 2003;317:21

17. Solenov D, Fedichkin L. Nonunitary quantum walks on hypercycles. Phys Rev A. 2006;73:012313.

18. Alessandro DD, Parlangeli G, Albertini F. Correlation between inflammatory factors and mood symptoms in bipolar disorder. J Phys A. 2007:40:14447.

19. Leung G, Knott P, Bailey J, Kendon V. Coined quantum walks on percolation graphs. New J Phys. 2010;12:123018.

20. Kollár B, Kiss T, Novotný J, Jex I. Asymptotic dynamics of coined quantum walks on percolation graphs. Phys Rev Lett. 2012:108:230505.

21. Kollár B, Novotný J, Kiss T, Jex I. Percolation induced effects in two-dimensional coined quantum walks: analytic asymptotic solutions. New J Phys. 2014;16:023002.

22. Douglas BL, Wang JB. A classical approach to the graph isomorphism problem using quantum walks. J Phys A. 2008:41:075303.

23. Breuer HP, Petruccione F. The theory of open quantum systems. Oxford: Oxford University Press: 2007.

24. Lindblad G. Generators of quantum dynamical semigroups. Commun Math Phys. 1976;48:119.

25. Yin Y, Katsanos DE, Evangelou SN. Quantum walks on a random environment. Phys Rev A. 2008;77:022302.

26. Schreiber A, Cassemiro KN, Potocek V, Gabris A, Jex I, Silberhorn C. Decoherence and disorder in QuantumWalks: from ballistic spread to localization. Phys Rev Lett. 2011;106:180403.

27. Mulken O, Blumen A. Continuous-time quantum walks: models for coherent transport on complex networks. Phys Rep. 2011;502:37.

28. Keating JP, Linden N, Matthews JCF, Winter A. Localization and its consequences for quantum walk algorithms and quantum communication. Phys Rev A. 2007;76:012315

29. Lamata L, Parra-Rodriguez A, Sanz M, Solano E. Digital-analog quantum simulations with superconducting circuits. Adv Phys X. 2018;3:1457981. 
30. Du YM, Lu LH, Li YQ. A rout to protect quantum gates constructed via quantum walks from noises. Sci Rep. 2018:8:7117.

31. Daras S, Gresch A, Groiseau C, Wimberger W, Summy GS. Quantum walk in momentum space with a Bose-Einstein condensate. Phys Rev Lett. 2018;121:070402.

32. Yan ZG, Zhang YR et al. Strongly correlated quantum walks with a 12-qubit superconducting processor. Science. 2019;364:753-6

33. Peruzzo A, Lobino M et al. Quantum walks of correlated photons. Science. 2010;329:1500.

34. Gou W, Chen T et al. Tunable nonreciprocal quantum transport through a dissipative Aharonov-Bohm ring in ultracold atoms. Phys Rev Lett. 2020;124:070402.

35. Broome MA, Fedrizzi A et al. Discrete single-photon quantum walks with tunable decoherence. Phys Rev Lett. 2010;104:153602.

Submit your manuscript to a SpringerOpen ${ }^{\circ}$ journal and benefit from:

- Convenient online submission

- Rigorous peer review

- Open access: articles freely available online

- High visibility within the field

- Retaining the copyright to your article

Submit your next manuscript at $\gg$ springeropen.com 